\title{
Improving ethylene glycol transport properties by caffeine - thermodynamic and computational evidence
}

Milan Vraneš ${ }^{1}$, Ivona Radović ${ }^{2}$, Siniša Bikić ${ }^{3}$, Aleksandar Tot $^{1 *}$, Mirjana Kijevčanin ${ }^{2}$, Milana Zarić $^{4}$, Teona Teodora Borović ${ }^{1}$, Snežana Papović ${ }^{1}$

${ }^{1}$ Faculty of Sciences, Department of Chemistry, Biochemistry and Environmental Protection,

University of Novi Sad, Trg Dositeja Obradovića 3, 21000 Novi Sad, Serbia

${ }^{2}$ Faculty of Technology and Metallurgy, University of Belgrade, Karnegijeva 4, 11120 Belgrade, Serbia

${ }^{3}$ Faculty of Technical Sciences, University of Novi Sad, Trg Dositeja Obradovića 6, 21000 Novi Sad, Serbia

${ }^{4}$ University of Belgrade - Institute of Chemistry, Technology and Metallurgy -National Institute of the Republic of Serbia Njegoševa 12 , 11120 Belgrade, Serbia

Table S1. Provenance, purity, and structure of the used chemicals.

${ }^{*}$ Corresponding Author: Tel: +381 21485 2751; Fax: +381 21454 065; E-mail: aleksandar.tot@dh.uns.ac.rs 


\begin{tabular}{ccccc}
\hline Chemical Name & Source & $\begin{array}{c}\text { CAS } \\
\text { Number }\end{array}$ & $\begin{array}{c}\text { Mass Fraction } \\
\text { Purity }\end{array}$ & Structure \\
\hline \multirow{2}{*}{ Caffeine } & $\begin{array}{c}\text { Sigma- } \\
\text { Aldrich }\end{array}$ & $58-08-2$ & $\geq 0.99$ \\
\hline Ehylene glycol & $\begin{array}{c}\text { Sigma- } \\
\text { Aldrich }\end{array}$ & $107-21-1$ & $\geq 0.99$ & \\
\hline
\end{tabular}


Table S2. Molality, $(m)$ and density, $(\rho)$ of caffeine + ethylene glycol solutions in the temperature range from $T=(288.15$ to 343.15$) \mathrm{K}$ at $p=1 \cdot 10^{5} \mathrm{~Pa}$.

\begin{tabular}{|c|c|c|c|c|c|c|c|c|c|c|c|c|}
\hline \multirow{2}{*}{$\begin{array}{c}\begin{array}{c}m / \\
\left(\mathrm{mol} \cdot \mathrm{kg}^{-1}\right)\end{array} \\
T /(\mathrm{K})\end{array}$} & \multicolumn{12}{|c|}{$\rho /\left(\mathrm{g} \cdot \mathrm{cm}^{-3}\right)$} \\
\hline & 288.15 & 293.15 & 298.15 & 303.15 & 308.15 & 313.15 & 318.15 & 323.15 & 328.15 & 333.15 & 338.15 & 343.15 \\
\hline 0.0000 & 1.116743 & 1.113266 & 1.109773 & 1.106266 & 1.102743 & 1.099201 & 1.095640 & 1.092056 & 1.088449 & 1.084817 & 1.081158 & 1.077645 \\
\hline 0.0090 & 1.117059 & 1.113582 & 1.110089 & 1.106580 & 1.103057 & 1.099515 & 1.095950 & 1.092362 & 1.088753 & 1.085115 & 1.081453 & 1.077933 \\
\hline 0.0181 & 1.117377 & 1.113898 & 1.110403 & 1.106892 & 1.103371 & 1.099828 & 1.096260 & 1.092666 & 1.089055 & 1.085412 & 1.081746 & 1.078219 \\
\hline 0.0268 & 1.117680 & 1.114199 & 1.110698 & 1.107185 & 1.103666 & 1.100122 & 1.096552 & 1.092952 & 1.089339 & 1.085690 & 1.082021 & 1.078488 \\
\hline 0.0358 & 1.117989 & 1.114504 & 1.110998 & 1.107482 & 1.103966 & 1.100422 & 1.096848 & 1.093241 & 1.089628 & 1.085971 & 1.082300 & 1.078761 \\
\hline 0.0461 & 1.118345 & 1.114855 & 1.111342 & 1.107823 & 1.104312 & 1.100766 & 1.097190 & 1.093575 & 1.089960 & 1.086295 & 1.082621 & 1.079075 \\
\hline 0.0542 & 1.118621 & 1.115126 & 1.111606 & 1.108084 & 1.104577 & 1.101030 & 1.097451 & 1.093829 & 1.090213 & 1.086542 & 1.082866 & 1.079315 \\
\hline
\end{tabular}

Standard uncertainties are: $u(\rho)=8.2 \cdot 10^{-5} \mathrm{~g} \cdot \mathrm{cm}^{-3}, u(m)=3.5 \cdot 10^{-4} \mathrm{~mol} \cdot \mathrm{kg}^{-1}, u(T)=0.01 \mathrm{~K}$;

Relative standard uncertainty: $u_{\mathrm{r}}(p)=0.015$. 
Table S3. Fitting parameters of the plot representing density as a function of the temperature (eq. S1) for each caffeine molality $(m)$, the standard deviation $(\sigma)$ of the fit with the regression coefficient $\left(R^{2}\right)$.

\begin{tabular}{ccccc}
\hline$m /\left(\mathrm{mol} \cdot \mathrm{kg}^{-1}\right)$ & $b_{\mathrm{o}} /\left(\mathrm{g} \cdot \mathrm{cm}^{-3}\right)$ & $b_{1} \cdot 10^{6} /\left(\mathrm{g} \cdot \mathrm{cm}^{-3} \cdot \mathrm{K}^{-1}\right)$ & $\sigma$ & $R^{2}$ \\
\hline 0.0000 & 1.32212 & -7.122 & 0.00241 & 0.99994 \\
0.0090 & 1.32258 & -7.127 & 0.00332 & 0.99994 \\
0.0181 & 1.32306 & -7.132 & 0.00704 & 0.99993 \\
0.0268 & 1.32352 & -7.138 & 0.01539 & 0.99993 \\
0.0358 & 1.32400 & -7.144 & 0.01765 & 0.99993 \\
0.0461 & 1.32455 & -7.150 & 0.02417 & 0.99993 \\
0.0542 & 1.32499 & -7.156 & 0.03074 & 0.99992 \\
\hline
\end{tabular}

The coefficients obtained from equation S1:

$\rho=b_{\mathrm{o}}+b_{1} T$

(S1),

where $b_{\mathrm{o}}$ is intercept, and $b_{1}$ is slope presented in Table S3.

Table S4. Fitting parameters of the plot representing density as a function of the caffeine molality $(m)$ (eq. S2), for each temperature, the standard deviation $(\sigma)$ of the fit with the regression coefficient $\left(R^{2}\right)$.

\begin{tabular}{ccccc}
\hline$T /(\mathrm{K})$ & $c_{0} \cdot 10^{-2} /\left(\mathrm{g} \cdot \mathrm{cm}^{-3}\right)$ & $c_{1} /\left(\mathrm{g} \cdot \mathrm{kg} \cdot \mathrm{cm}^{-3} \cdot \mathrm{mol}^{-1}\right)$ & $\sigma$ & $R^{2}$ \\
\hline 288.15 & 3.465 & 1.11675 & 0.00147 & 0.99999 \\
293.15 & 3.431 & 1.11327 & 0.00182 & 0.99997 \\
298.15 & 3.379 & 1.10978 & 0.00167 & 0.99993 \\
303.15 & 3.351 & 1.10628 & 0.00158 & 0.99992 \\
308.15 & 3.382 & 1.10275 & 0.00149 & 0.99995 \\
313.15 & 3.372 & 1.09921 & 0.00180 & 0.99994 \\
318.15 & 3.340 & 1.09565 & 0.00174 & 0.99995 \\
323.15 & 3.269 & 1.09207 & 0.00168 & 0.99993 \\
328.15 & 3.253 & 1.08846 & 0.00173 & 0.99993 \\
333.15 & 3.180 & 1.08483 & 0.00168 & 0.99991 \\
338.15 & 3.149 & 1.08117 & 0.00175 & 0.99992 \\
343.15 & 3.079 & 1.07766 & 0.00159 & 0.99993 \\
\hline
\end{tabular}

The coefficients obtained from equation S2:

$\rho=c_{\mathrm{o}}+c_{1} m$

(S2),

where $c_{0}$ is intercept, and $c_{1}$ is slope presented in Table S4. 
Table S5. Values of thermal expansion coefficient, $\left(\alpha_{\mathrm{p}}\right)$, for caffeine + ethylene glycol solutions for each caffeine molality $(m)$ in the temperature range from $T=$ (288.15 to 343.15$) \mathrm{K}$ at $p=1 \cdot 10^{5} \mathrm{~Pa}$.

\begin{tabular}{|c|c|c|c|c|c|c|c|c|c|c|c|c|}
\hline \multirow{2}{*}{$\begin{array}{c}\begin{array}{c}m / \\
\left(\mathrm{mol} \cdot \mathrm{kg}^{-1}\right)\end{array} \\
T /(\mathrm{K})\end{array}$} & \multicolumn{12}{|c|}{$\alpha_{\mathrm{p}} \cdot 10^{4} /\left(\mathrm{K}^{-1}\right)$} \\
\hline & 288.15 & 293.15 & 298.15 & 303.15 & 308.15 & 313.15 & 318.15 & 323.15 & 328.15 & 333.15 & 338.15 & 343.15 \\
\hline 0.0000 & 6.38 & 6.40 & 6.42 & 6.44 & 6.46 & 6.48 & 6.50 & 6.52 & 6.54 & 6.57 & 6.59 & 6.61 \\
\hline 0.0090 & 6.38 & 6.40 & 6.42 & 6.44 & 6.46 & 6.48 & 6.50 & 6.52 & 6.55 & 6.57 & 6.59 & 6.61 \\
\hline 0.0181 & 6.38 & 6.40 & 6.42 & 6.44 & 6.46 & 6.48 & 6.51 & 6.53 & 6.55 & 6.57 & 6.59 & 6.61 \\
\hline 0.0268 & 6.39 & 6.41 & 6.43 & 6.45 & 6.47 & 6.49 & 6.51 & 6.53 & 6.55 & 6.57 & 6.60 & 6.62 \\
\hline 0.0358 & 6.39 & 6.41 & 6.43 & 6.45 & 6.47 & 6.49 & 6.51 & 6.53 & 6.56 & 6.58 & 6.60 & 6.62 \\
\hline 0.0461 & 6.39 & 6.41 & 6.43 & 6.45 & 6.47 & 6.50 & 6.52 & 6.54 & 6.56 & 6.58 & 6.60 & 6.63 \\
\hline 0.0542 & 6.40 & 6.42 & 6.44 & 6.46 & 6.48 & 6.50 & 6.52 & 6.54 & 6.56 & 6.59 & 6.61 & 6.63 \\
\hline
\end{tabular}


Table S6. Apparent molar volume, $\left(V_{\phi}\right)$, partial molar volume of ethylene glycol $\left(\bar{V}_{1}\right)$ and partial molar volume of caffeine, $\left(\bar{V}_{2}\right)$, in caffeine + ethylene glycol mixture in the temperature range from $T=(288.15$ to 343.15$) \mathrm{K}$ at $p=1 \cdot 10^{5} \mathrm{~Pa}$.

\begin{tabular}{|c|c|c|c|c|c|c|c|c|c|c|c|c|}
\hline $\begin{array}{c}m / \\
\left(m o l \cdot \mathrm{kg}^{-1}\right)\end{array}$ & \multicolumn{12}{|c|}{$V_{\phi} /\left(\mathrm{cm}^{3} \cdot \mathrm{mol}^{-1}\right)$} \\
\hline$T /(\mathrm{K})$ & 288.15 & 293.15 & 298.15 & 303.15 & 308.15 & 313.15 & 318.15 & 323.15 & 328.15 & 333.15 & 338.15 & 343.15 \\
\hline 0.0090 & 145.60 & 145.96 & 146.33 & 146.89 & 147.27 & 147.65 & 148.40 & 149.17 & 149.76 & 150.73 & 151.43 & 152.50 \\
\hline 0.0181 & 145.59 & 146.05 & 146.51 & 147.06 & 147.35 & 147.78 & 148.49 & 149.35 & 149.93 & 150.86 & 151.61 & 152.68 \\
\hline 0.0268 & 145.68 & 146.16 & 146.78 & 147.34 & 147.59 & 148.04 & 148.71 & 149.61 & 150.20 & 151.15 & 151.88 & 152.93 \\
\hline 0.0358 & 145.71 & 146.25 & 146.92 & 147.51 & 147.73 & 148.16 & 148.85 & 149.79 & 150.33 & 151.34 & 152.05 & 153.08 \\
\hline 0.0461 & 145.71 & 146.31 & 147.03 & 147.63 & 147.79 & 148.25 & 148.91 & 149.88 & 150.43 & 151.45 & 152.15 & 153.18 \\
\hline 0.0542 & 145.77 & 146.41 & 147.19 & 147.79 & 147.93 & 148.39 & 149.06 & 150.05 & 150.60 & 151.63 & 152.32 & 153.33 \\
\hline $\begin{array}{c}m / \\
\left(\mathrm{mol} \cdot \mathrm{kg}^{-1}\right)\end{array}$ & \multicolumn{12}{|c|}{$\bar{V}_{1} /\left(\mathrm{cm}^{3} \cdot \mathrm{mol}^{-1}\right)$} \\
\hline 0.0090 & 55.5793 & 55.7528 & 55.9282 & 56.1055 & 56.2848 & 56.4661 & 56.6497 & 56.8356 & 57.0239 & 57.2148 & 57.4085 & 57.5956 \\
\hline 0.0181 & 55.5792 & 55.7527 & 55.9279 & 56.1052 & 56.2845 & 56.4659 & 56.6494 & 56.8352 & 57.0236 & 57.2145 & 57.4081 & 57.5953 \\
\hline 0.0268 & 55.5791 & 55.7525 & 55.9275 & 56.1048 & 56.2842 & 56.4655 & 56.6491 & 56.8349 & 57.0232 & 57.2141 & 57.4077 & 57.5950 \\
\hline 0.0358 & 55.5790 & 55.7522 & 55.9271 & 56.1043 & 56.2839 & 56.4651 & 56.6488 & 56.8344 & 57.0228 & 57.2136 & 57.4073 & 57.5945 \\
\hline 0.0461 & 55.5789 & 55.7519 & 55.9265 & 56.1036 & 56.2834 & 56.4646 & 56.6483 & 56.8338 & 57.0222 & 57.2129 & 57.4066 & 57.5939 \\
\hline 0.0542 & 55.5788 & 55.7516 & 55.9259 & 56.1031 & 56.2830 & 56.4641 & 56.6479 & 56.8332 & 57.0217 & 57.2124 & 57.4061 & 57.5934 \\
\hline $\begin{array}{c}m / \\
\left(\mathrm{mol} \cdot \mathrm{kg}^{-1}\right)\end{array}$ & \multicolumn{12}{|c|}{$\bar{V}_{2} /\left(\mathrm{cm}^{3} \cdot \mathrm{mol}^{-1}\right)$} \\
\hline 0.0090 & 145.66 & 146.12 & 146.63 & 147.20 & 147.50 & 147.91 & 148.64 & 149.48 & 150.05 & 151.05 & 151.74 & 152.79 \\
\hline 0.0181 & 145.68 & 146.26 & 146.93 & 147.51 & 147.68 & 148.15 & 148.82 & 149.78 & 150.34 & 151.31 & 152.04 & 153.08 \\
\hline 0.0268 & 145.78 & 146.43 & 147.29 & 147.88 & 148.00 & 148.49 & 149.11 & 150.13 & 150.69 & 151.69 & 152.41 & 153.43 \\
\hline 0.0358 & 145.83 & 146.56 & 147.51 & 148.13 & 148.19 & 148.68 & 149.31 & 150.40 & 150.91 & 151.97 & 152.66 & 153.65 \\
\hline 0.0461 & 145.85 & 146.65 & 147.70 & 148.34 & 148.33 & 148.84 & 149.44 & 150.57 & 151.08 & 152.17 & 152.85 & 153.83 \\
\hline 0.0542 & 145.92 & 146.78 & 147.91 & 148.56 & 148.51 & 149.03 & 149.63 & 150.80 & 151.31 & 152.40 & 153.07 & 154.04 \\
\hline
\end{tabular}

ancertainties are: $u(\rho)=8.2 \cdot 10^{-5} \mathrm{~g} \cdot \mathrm{cm}^{-3}, u(m)=3.5 \cdot 10^{-4} \mathrm{~mol} \cdot \mathrm{kg}^{-1}, u(T)=0.01 \mathrm{~K}, u\left(V_{\phi}\right)=5.6 \cdot 10^{-3} \mathrm{~cm}^{3} \cdot \mathrm{mol}^{-1} ; u\left(\bar{V}_{2}\right)=6.9 \cdot 10^{-3} \mathrm{~cm}^{3} \cdot \mathrm{mol}^{-1} ;$

Relative standard uncertainty: $u_{\mathrm{r}}(p)=0.015$.

*calculated by eq (S3)

** calculated by eq (S4 and S5) 
From the experimental densities the apparent molar volumes, $\left(V_{\phi}\right)$, were calculated using the equation:

$V_{\phi}=\frac{1000\left(\rho_{1}-\rho\right)}{m \rho \rho_{1}}+\frac{M_{2}}{\rho}$

The partial molar volumes of ethylene glycol $\left(\bar{V}_{1}\right)$ and caffeine $\left(\bar{V}_{2}\right)$ were calculated using the equations:

$\bar{V}_{1}=\frac{M_{1}}{\rho_{1}}-\frac{M_{1} m^{3 / 2}}{2000}\left(\frac{\partial V_{\phi}}{\partial \sqrt{m}}\right)_{T, p, n_{2}}$

$\bar{V}_{2}=\frac{\sqrt{m}}{2}\left(\frac{\partial V_{\phi}}{\partial \sqrt{m}}\right)_{T, p, n_{1}}+V_{\phi}$

In equations S3 to S5 $m\left(\mathrm{~mol} \cdot \mathrm{kg}^{-1}\right)$ represents molality of caffeine, $\rho\left(\mathrm{g} \cdot \mathrm{cm}^{-3}\right)$ the experimental density of the solution, $\rho_{1}\left(\mathrm{~g} \cdot \mathrm{cm}^{-3}\right)$ the experimental density of the ethylene glycol, $M_{1}$ and $M_{2}\left(\mathrm{~g} \cdot \mathrm{mol}^{-1}\right)$ are molar masses of ethylene glycol and of caffeine, respectively. Calculated values of the apparent molar volumes and partial molar volumes for investigated systems are given in the Table S6. 
Table S7. Fitting coefficients $a_{0}, a_{1}$ and $a_{2}$ of apparent molar volumes, $\left(V_{\phi}\right)$, of the caffeine + ethylene glycol mixtures (eq. 4), with the regression coefficient $\left(R^{2}\right)$.

\begin{tabular}{cccc}
\hline$a_{\mathrm{o}} /\left(\mathrm{cm}^{3} \cdot \mathrm{mol}^{-1}\right)$ & $a_{1} /\left(\mathrm{cm}^{3} \cdot \mathrm{mol}^{-1} \cdot \mathrm{K}^{-1}\right)$ & $a_{2} /\left(\mathrm{cm}^{3} \cdot \mathrm{mol}^{-1} \cdot \mathrm{K}^{-2}\right)$ & $R^{2}$ \\
\hline 261.05 & -0.8373 & -0.00151 & 0.9980 \\
\hline
\end{tabular}

Table S8. Values of the limiting apparent molar expansibilities, $\left(E_{\phi}^{0}\right)$ of the caffeine + ethylene glycol solutions (eq. 5) in the temperature range from $T=(288.15$ to 343.15$) \mathrm{K}$.

\begin{tabular}{|c|c|c|c|c|c|c|c|c|c|c|c|c|}
\hline \multicolumn{13}{|c|}{$E_{\phi}^{\mathrm{o}} /\left(\mathrm{cm}^{3} \cdot \mathrm{mol}^{-1} \cdot \mathrm{K}^{-1}\right)$} \\
\hline$T /(\mathrm{K})$ & 288.15 & 293.15 & 298.15 & 303.15 & 308.15 & 313.15 & 318.15 & 323.15 & 328.15 & 333.15 & 338.15 & 343.15 \\
\hline & 0.02715 & 0.04215 & 0.05716 & 0.07214 & 0.08715 & 0.1022 & 0.1172 & 0.1322 & 0.1473 & 0.1622 & 0.1771 & 0.1922 \\
\hline
\end{tabular}


Table S9. Viscosity of caffeine + ethylene glycol solutions in the temperature range from $T=(288.15$ to 343.15$) \mathrm{K}$ at $p=1 \cdot 10^{5} \mathrm{~Pa}$.

\begin{tabular}{|c|c|c|c|c|c|c|c|c|c|c|c|c|}
\hline \multirow{2}{*}{$\begin{array}{c}\begin{array}{c}m / \\
\left(\mathrm{mol} \cdot \mathrm{kg}^{-1}\right)\end{array} \\
T /(\mathrm{K})\end{array}$} & \multicolumn{12}{|c|}{$\eta /(\mathrm{mPa} \cdot \mathrm{s})$} \\
\hline & 288.15 & 293.15 & 298.15 & 303.15 & 308.15 & 313.15 & 318.15 & 323.15 & 328.15 & 333.15 & 338.15 & 343.15 \\
\hline 0.0000 & 26.343 & 20.857 & 16.909 & 13.831 & 11.444 & 9.590 & 8.126 & 6.933 & 5.973 & 5.188 & 4.535 & 4.016 \\
\hline 0.0090 & 26.253 & 20.794 & 16.863 & 13.792 & 11.410 & 9.550 & 8.092 & 6.902 & 5.956 & 5.172 & 4.514 & 3.994 \\
\hline 0.0181 & 26.186 & 20.751 & 16.800 & 13.766 & 11.364 & 9.528 & 8.070 & 6.890 & 5.923 & 5.144 & 4.495 & 3.978 \\
\hline 0.0268 & 26.136 & 20.688 & 16.758 & 13.717 & 11.341 & 9.492 & 8.048 & 6.866 & 5.903 & 5.130 & 4.479 & 3.962 \\
\hline 0.0358 & 26.047 & 20.636 & 16.714 & 13.674 & 11.300 & 9.464 & 8.019 & 6.833 & 5.886 & 5.102 & 4.460 & 3.941 \\
\hline 0.0461 & 25.981 & 20.570 & 16.656 & 13.626 & 11.272 & 9.426 & 7.982 & 6.805 & 5.859 & 5.087 & 4.439 & 3.925 \\
\hline 0.0542 & 25.939 & 20.526 & 16.628 & 13.590 & 11.231 & 9.403 & 7.965 & 6.785 & 5.845 & 5.061 & 4.416 & 3.910 \\
\hline
\end{tabular}

Standard uncertainties: $u(T)=0.01 \mathrm{~K}, u(m)=3.5 \cdot 10^{-4} \mathrm{~mol} \cdot \mathrm{kg}^{-1}$,

Relative standard uncertainties: $u_{r}(\eta)=0.002, u_{\mathrm{r}}(p)=0.015$. 
Table S10. Values of viscosity, shear stress, shear rate and revolution per minute of spindle (RPM), at molality of caffeine $m=0.0542 \mathrm{~mol} \cdot \mathrm{kg}^{-1}$ and at $T=298.15 \mathrm{~K}$ and $p=1 \cdot 10^{5} \mathrm{~Pa}$.

\begin{tabular}{cccc}
\hline RPM & $\eta /(\mathrm{mPa} \cdot \mathrm{s})$ & Shear stress $/\left(\mathrm{N} \cdot \mathrm{m}^{-2}\right)$ & Shear rate $/\left(\mathrm{s}^{-1}\right)$ \\
\hline 105 & 16.59 & 2.340 & 138.6 \\
120 & 16.62 & 2.681 & 158.4 \\
135 & 16.71 & 3.030 & 178.2 \\
140 & 16.74 & 3.150 & 184.8 \\
150 & 16.68 & 3.363 & 198.0 \\
160 & 16.79 & 3.607 & 211.2 \\
\hline
\end{tabular}

Standard uncertainties: $u(T)=0.01 \mathrm{~K}$;

Relative standard uncertainties: $u_{\mathrm{r}}(\eta)=0.002, u_{\mathrm{r}}(p)=0.015$. 
Table S11. Thermodynamics parameters of viscous flow values in the temperature range from $T=(288.15$ to 343.15$) \mathrm{K}$.

\begin{tabular}{|c|c|c|c|c|c|c|c|c|c|c|c|c|}
\hline \multirow{2}{*}{ Parameters } & \multicolumn{12}{|c|}{$T /(\mathrm{K})$} \\
\hline & 288.15 & 293.15 & 298.15 & 303.15 & 308.15 & 313.15 & 318.15 & 323.15 & 328.15 & 333.15 & 338.15 & 343.15 \\
\hline \multicolumn{13}{|c|}{ Caffeine + ethylene glycol } \\
\hline $\bar{V}_{2}^{\mathrm{o}} /\left(\mathrm{cm}^{3} \cdot \mathrm{mol}^{-1}\right)$ & 145.46 & 145.64 & 145.72 & 146.23 & 146.76 & 147.10 & 147.90 & 148.53 & 149.17 & 150.05 & 150.79 & 151.91 \\
\hline $\bar{V}_{1}^{\mathrm{o}} /\left(\mathrm{cm}^{3} \cdot \mathrm{mol}^{-1}\right)$ & 55.58 & 55.75 & 55.93 & 56.11 & 56.28 & 56.47 & 56.65 & 56.84 & 57.02 & 57.21 & 57.41 & 57.60 \\
\hline$\Delta \mu_{1}^{\mathrm{o} \neq} /\left(\mathrm{kJ} \cdot \mathrm{mol}^{-1}\right)$ & 19.66 & 19.44 & 19.26 & 19.09 & 18.92 & 18.78 & 18.65 & 18.53 & 18.41 & 18.31 & 18.22 & 18.15 \\
\hline$\Delta \mu_{2}^{\mathrm{o} \neq} /\left(\mathrm{kJ} \cdot \mathrm{mol}^{-1}\right)$ & 12.98 & 11.83 & 10.88 & 9.44 & 9.21 & 8.14 & 7.47 & 5.59 & 5.01 & 2.30 & 1.39 & 1.08 \\
\hline \multicolumn{13}{|c|}{ Caffeine + water* } \\
\hline $\bar{V}_{2}^{\mathrm{o}} /\left(\mathrm{cm}^{3} \cdot \mathrm{mol}^{-1}\right)$ & 141.05 & 141.99 & 142.93 & 143.59 & 144.45 & 145.20 & - & - & - & - & - & - \\
\hline $\bar{V}_{1}^{\mathrm{o}} /\left(\mathrm{cm}^{3} \cdot \mathrm{mol}^{-1}\right)$ & 18.02 & 18.03 & 18.05 & 18.07 & 18.09 & 18.12 & - & - & - & - & - & - \\
\hline$\Delta \mu_{1}^{\mathrm{o} \neq} /\left(\mathrm{kJ} \cdot \mathrm{mol}^{-1}\right)$ & 9.60 & 9.44 & 9.29 & 9.16 & 9.04 & 8.93 & - & - & - & - & - & - \\
\hline$\Delta \mu_{2}^{\mathrm{o} \neq} /\left(\mathrm{kJ} \cdot \mathrm{mol}^{-1}\right)$ & 77.7 & 79.3 & 82.1 & 84.2 & 86.4 & 88.0 & - & - & - & - & - & - \\
\hline
\end{tabular}

*Results taken from work of Vraneš et al. [69] 
Table S12. Refractive index, $\left(n_{\mathrm{D}}\right)$ of caffeine + ethylene glycol solutions in the temperature range from $T=(288.15$ to 343.15$) \mathrm{K}$ at $p=1 \cdot 10^{5} \mathrm{~Pa}$.

\begin{tabular}{|c|c|c|c|c|c|c|c|c|c|c|c|c|}
\hline $\begin{array}{c}m / \\
\left(\mathrm{mol} \cdot \mathrm{kg}^{-1}\right)\end{array}$ & \multicolumn{12}{|c|}{$n_{\mathrm{D}}$} \\
\hline$T /(\mathrm{K})$ & 288.15 & 293.15 & 298.15 & 303.15 & 308.15 & 313.15 & 318.15 & 323.15 & 328.15 & 333.15 & 338.15 & 343.15 \\
\hline 0.0000 & 1.43335 & 1.43190 & 1.43046 & 1.42901 & 1.42751 & 1.42601 & 1.42450 & 1.42295 & 1.42138 & 1.41979 & 1.41824 & 1.41669 \\
\hline 0.0090 & 1.43358 & 1.43215 & 1.43070 & 1.42922 & 1.42772 & 1.42619 & 1.42466 & 1.42311 & 1.42153 & 1.41995 & 1.41842 & 1.41685 \\
\hline 0.0181 & 1.43378 & 1.43235 & 1.4309 & 1.42943 & 1.42791 & 1.42639 & 1.42486 & 1.42330 & 1.42173 & 1.42017 & 1.41862 & 1.41706 \\
\hline 0.0268 & 1.43398 & 1.43254 & 1.43109 & 1.42961 & 1.42810 & 1.42657 & 1.42501 & 1.42343 & 1.42188 & 1.42030 & 1.41877 & 1.41723 \\
\hline 0.0358 & 1.43420 & 1.43273 & 1.43127 & 1.42979 & 1.42830 & 1.42680 & 1.42520 & 1.42362 & 1.42205 & 1.42049 & 1.41896 & 1.41741 \\
\hline 0.0461 & 1.43445 & 1.43299 & 1.43153 & 1.43007 & 1.42854 & 1.42699 & 1.42541 & 1.42383 & 1.42227 & 1.42071 & 1.41918 & 1.41760 \\
\hline 0.0542 & 1.43464 & 1.43317 & 1.43171 & 1.43022 & 1.42870 & 1.42716 & 1.42556 & 1.42395 & 1.42242 & 1.42088 & 1.41934 & 1.41777 \\
\hline
\end{tabular}

Standard uncertainties are: $u\left(n_{\mathrm{D}}\right)=5.2 \cdot 10^{-4}, u(m)=3.5 \cdot 10^{-4} \mathrm{~mol} \cdot \mathrm{kg}^{-1}, u(T)=0.01 \mathrm{~K}$;

Relative standard uncertainty: $u_{\mathrm{r}}(p)=0.015$. 
Table S13. Fitting parameters of the plot representing refractive index as a function of the temperature (eq. S6) for each caffeine molality $(m)$, the standard deviation $(\sigma)$ of the fit with the regression coefficient $\left(R^{2}\right)$.

\begin{tabular}{ccccc}
\hline$m /\left(\mathrm{mol} \cdot \mathrm{kg}^{-1}\right)$ & $e_{\mathrm{o}}$ & $e_{1} /\left(\mathrm{K}^{-1}\right)$ & $\sigma$ & $R^{2}$ \\
\hline 0.0000 & 1.52100 & -3.037 & 0.00338 & 0.99986 \\
0.0090 & 1.52173 & -3.054 & 0.00327 & 0.99989 \\
0.0181 & 1.52188 & -3.052 & 0.00684 & 0.99990 \\
0.0268 & 1.52238 & -3.063 & 0.01289 & 0.99991 \\
0.0358 & 1.52268 & -3.066 & 0.01547 & 0.99990 \\
0.0461 & 1.52327 & -3.078 & 0.02071 & 0.99991 \\
0.0542 & 1.52357 & -3.081 & 0.03001 & 0.99993 \\
\hline
\end{tabular}

The coefficients obtained from equation S6:

$$
n_{\mathrm{D}}=e_{\mathrm{o}}+e_{1} T
$$

where $e_{\mathrm{o}}$ is intercept, and $e_{1}$ is slope from $\mathrm{S} 6$ equation, and presented in Table S13.

Table S14. Fitting parameters of the plot representing refractive index as a function of the caffeine molality $(m)$ (eq. S7), for each temperature, the standard deviation $(\sigma)$ of the fit with the regression coefficient $\left(R^{2}\right)$.

\begin{tabular}{ccccc}
\hline$T /(\mathrm{K})$ & $f_{\mathrm{o}}$ & $f_{\mathrm{l}} /\left(\mathrm{kg} \cdot \mathrm{mol}^{-1}\right)$ & $\sigma$ & $R^{2}$ \\
\hline 288.15 & 1.433 & 1.11675 & 0.02370 & 0.99988 \\
293.15 & 1.432 & 1.11327 & 0.02307 & 0.99945 \\
298.15 & 1.430 & 1.10978 & 0.02272 & 0.99949 \\
303.15 & 1.429 & 1.10628 & 0.02237 & 0.99937 \\
308.15 & 1.428 & 1.10275 & 0.02201 & 0.99987 \\
313.15 & 1.426 & 1.09921 & 0.02145 & 0.99951 \\
318.15 & 1.424 & 1.09565 & 0.01973 & 0.99973 \\
323.15 & 1.423 & 1.09207 & 0.01871 & 0.99927 \\
328.15 & 1.421 & 1.08846 & 0.01934 & 0.99959 \\
333.15 & 1.420 & 1.08483 & 0.02008 & 0.99920 \\
338.15 & 1.420 & 1.08117 & 0.00203 & 0.99976 \\
343.15 & 1.417 & 1.07766 & 0.01999 & 0.99968 \\
\hline
\end{tabular}


The coefficients obtained from equation (S7):

$$
n_{\mathrm{D}}=f_{\mathrm{o}}+f_{1} m
$$

where $f_{\mathrm{o}}$ is intercept, and $f_{1}$ is slope presented in Table S14.

Table S15. Refraction molar values, $\left(R_{\mathrm{m}}\right)$, of caffeine + ethylene glycol solutions in the temperature range from $T=(288.15$ to 343.15$) \mathrm{K}$ at $p=1 \cdot 10^{5}$ $\mathrm{Pa}$.

\begin{tabular}{|c|c|c|c|c|c|c|c|c|c|c|c|c|}
\hline \multirow{2}{*}{$\begin{array}{c}\begin{array}{c}m / \\
\left(\mathrm{mol} \cdot \mathrm{kg}^{-1}\right)\end{array} \\
T /(\mathrm{K})\end{array}$} & \multicolumn{12}{|c|}{$R_{\mathrm{m}} /\left(\mathrm{cm}^{3} \cdot \mathrm{mol}^{-1}\right)$} \\
\hline & 288.15 & 293.15 & 298.15 & 303.15 & 308.15 & 313.15 & 318.15 & 323.15 & 328.15 & 333.15 & 338.15 & 343.15 \\
\hline 0.0000 & 14.455 & 14.458 & 14.461 & 14.464 & 14.466 & 14.468 & 14.470 & 14.471 & 14.472 & 14.473 & 14.475 & 14.475 \\
\hline 0.0090 & 14.475 & 14.478 & 14.481 & 14.484 & 14.486 & 14.487 & 14.488 & 14.489 & 14.490 & 14.491 & 14.493 & 14.493 \\
\hline 0.0181 & 14.494 & 14.497 & 14.500 & 14.503 & 14.504 & 14.506 & 14.507 & 14.508 & 14.509 & 14.511 & 14.513 & 14.513 \\
\hline 0.0268 & 14.512 & 14.516 & 14.519 & 14.521 & 14.523 & 14.524 & 14.525 & 14.525 & 14.527 & 14.528 & 14.530 & 14.531 \\
\hline 0.0358 & 14.532 & 14.534 & 14.537 & 14.540 & 14.542 & 14.544 & 14.543 & 14.544 & 14.545 & 14.547 & 14.549 & 14.550 \\
\hline 0.0461 & 14.554 & 14.557 & 14.560 & 14.563 & 14.564 & 14.565 & 14.565 & 14.566 & 14.567 & 14.569 & 14.571 & 14.571 \\
\hline 0.0542 & 14.571 & 14.574 & 14.577 & 14.579 & 14.581 & 14.582 & 14.581 & 14.581 & 14.583 & 14.586 & 14.588 & 14.588 \\
\hline
\end{tabular}


a)

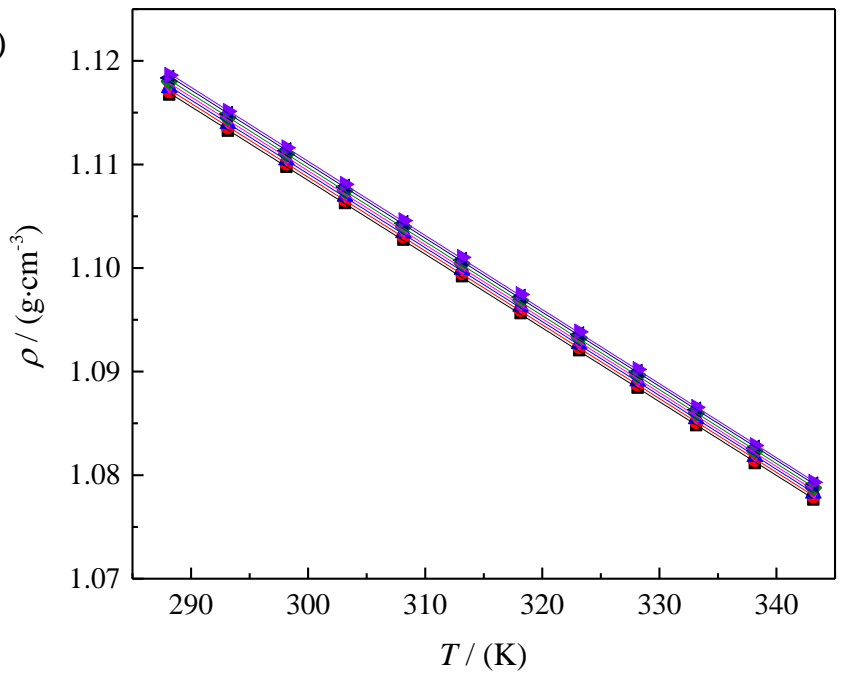

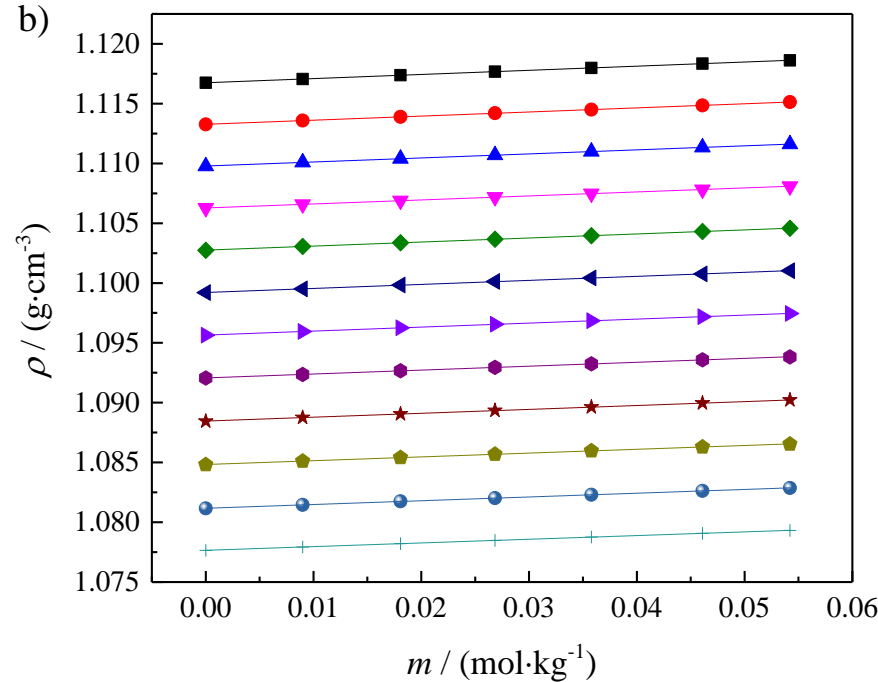

Figure S1. Variation of density, $(\rho)$, for caffeine + ethylene glycol solutions as a function of:

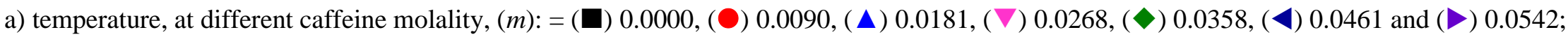
b) molality, at different temperatures, $(T):=(\mathbf{\square}) 288.15 ;(\bullet) 293.15 ;(\boldsymbol{\Delta}) 298.15 ;(\nabla) 303.15 ;(\diamond) 308.15 ;(\varangle) 313.15 ;(\nabla) 318.15 ;(\bullet) 323.15 ;(*)$ 328.15; ( 333.15 ; (๑) 338.15 and (+) $343.15 \mathrm{~K}$. The points represent experimental values while lines are fitts obtained using eqs. S1 and S2. 


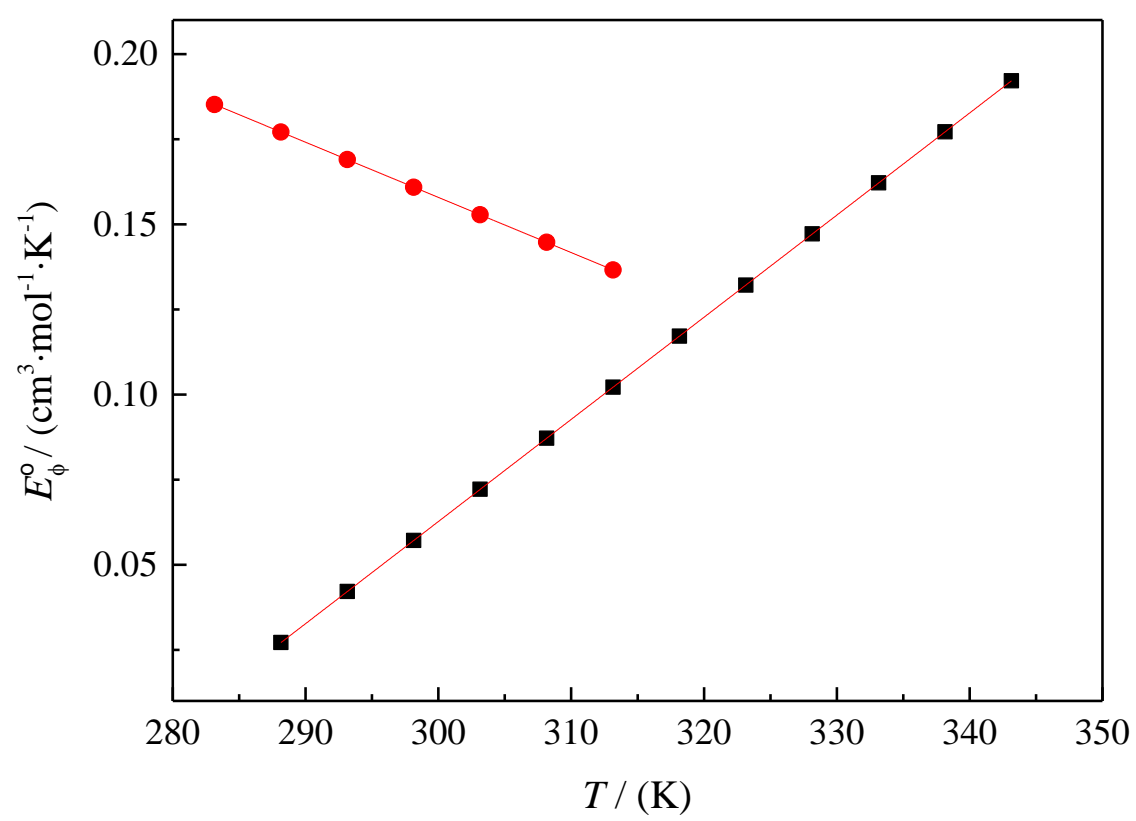

Figure S2. Variation of the limiting apparent molar expansibility, $\left(E_{\phi}^{\mathrm{o}}\right)$ of caffeine in: $(\bigcirc)$ aqueous solutions [64] and (ם) ethylene glycol solutions with a temperature. 
a)

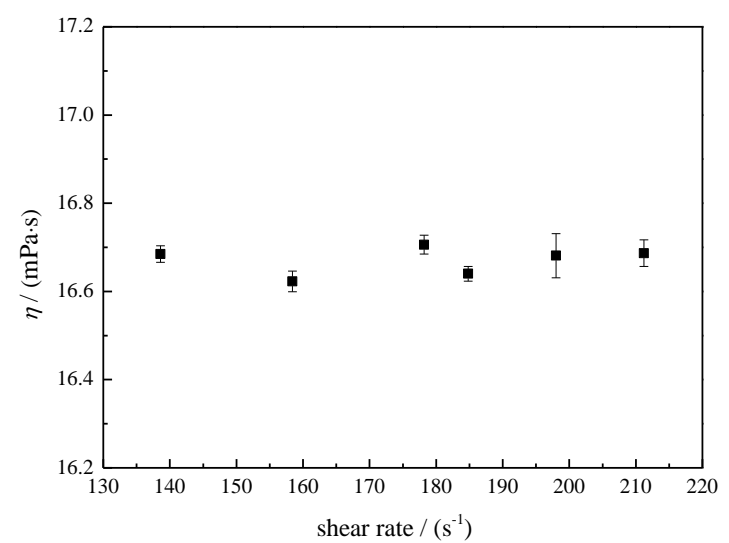

b)

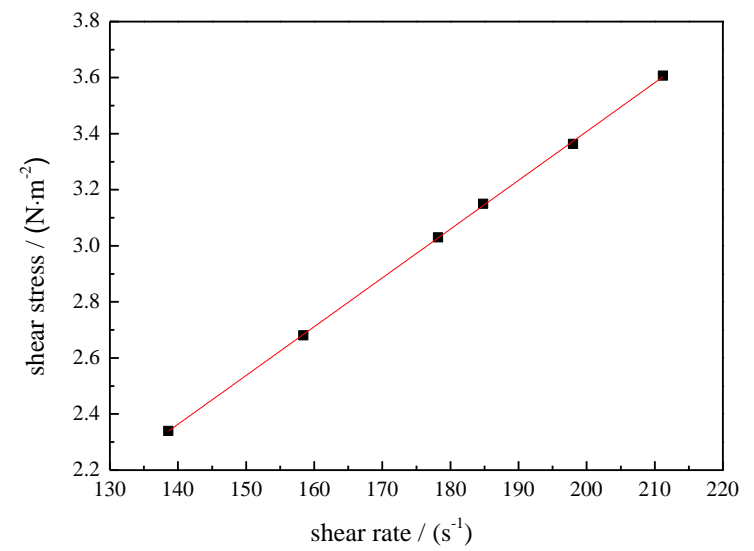

Figure S3. a) Changes of viscosity values with shear rate along with error bars, for caffeine + ethylene glycol solutions, at molality of caffeine $m=$ $0.0542 \mathrm{~mol} \cdot \mathrm{kg}^{-1}$ and at $T=298.15 \mathrm{~K}$ and b) flow curve, showing shear stress as a function of shear rate. 


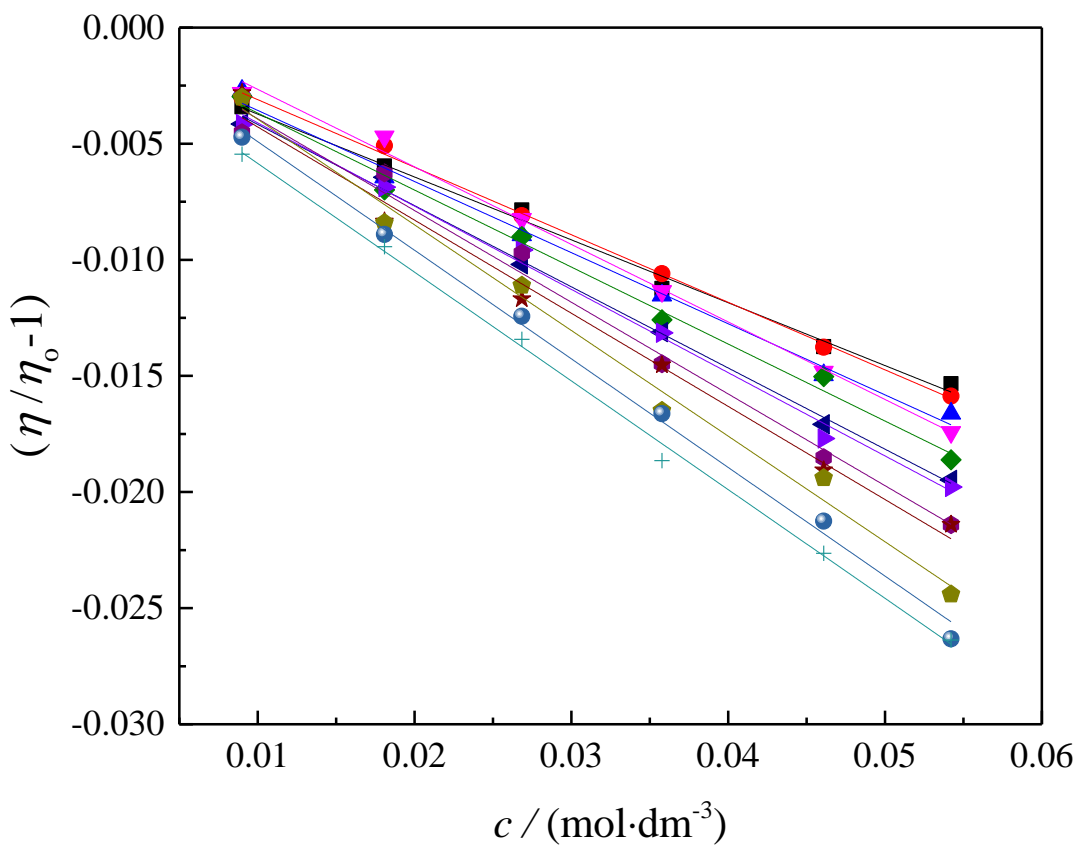

Figure S4. Plot of reduced viscosity, $\left(\frac{\eta}{\eta}-1\right)$, against concentration, $(c)$, of the caffeine + ethylene glycol mixture, at different temperatures: $T=($

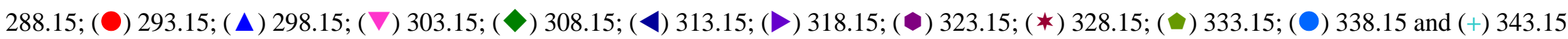
$\mathrm{K}$. The points represent experimental values and lines represent values calculated from eq. (7). 
a)
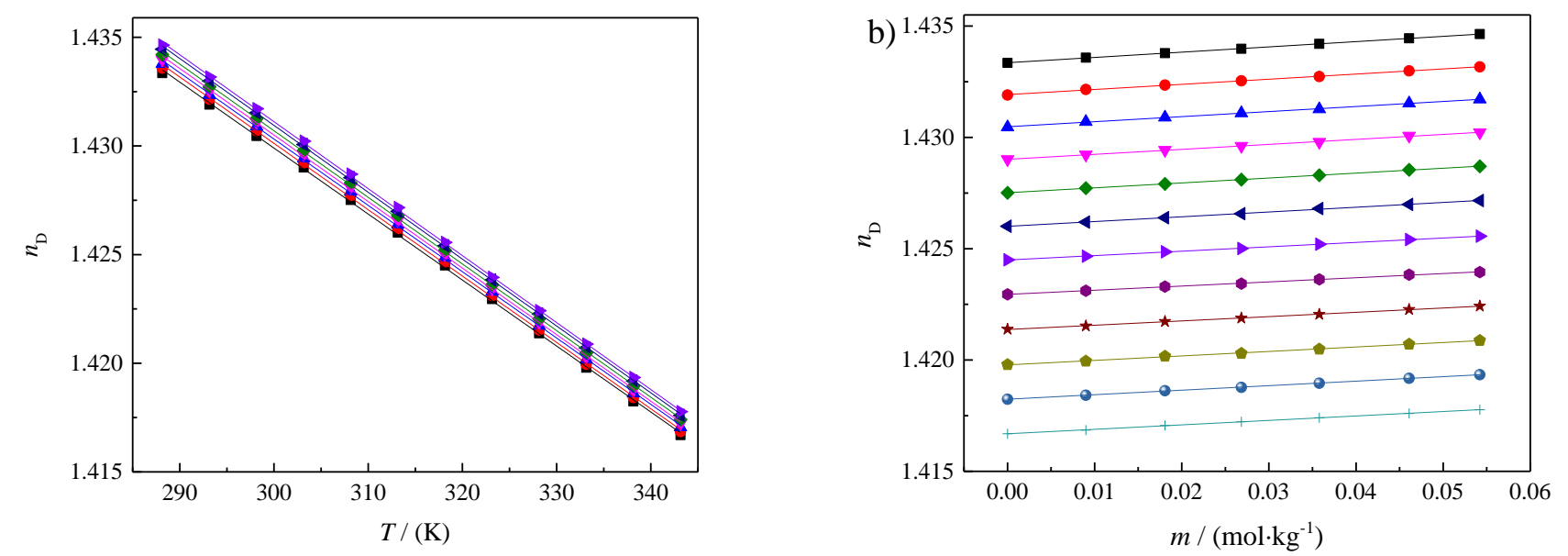

Figure S5. Variation of refractive index, $\left(n_{\mathrm{D}}\right)$, for caffeine + ethylene glycol solutions as a function of:

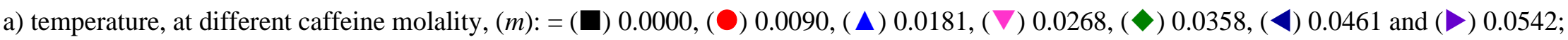

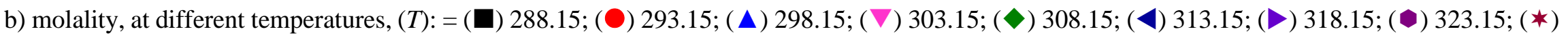
328.15; ( $(\bullet) 333.15 ;(\bullet) 338.15$ and (+) $343.15 \mathrm{~K}$. The points represent experimental values, while the lines represents fitts obtaned by eq. S6 and S7. 


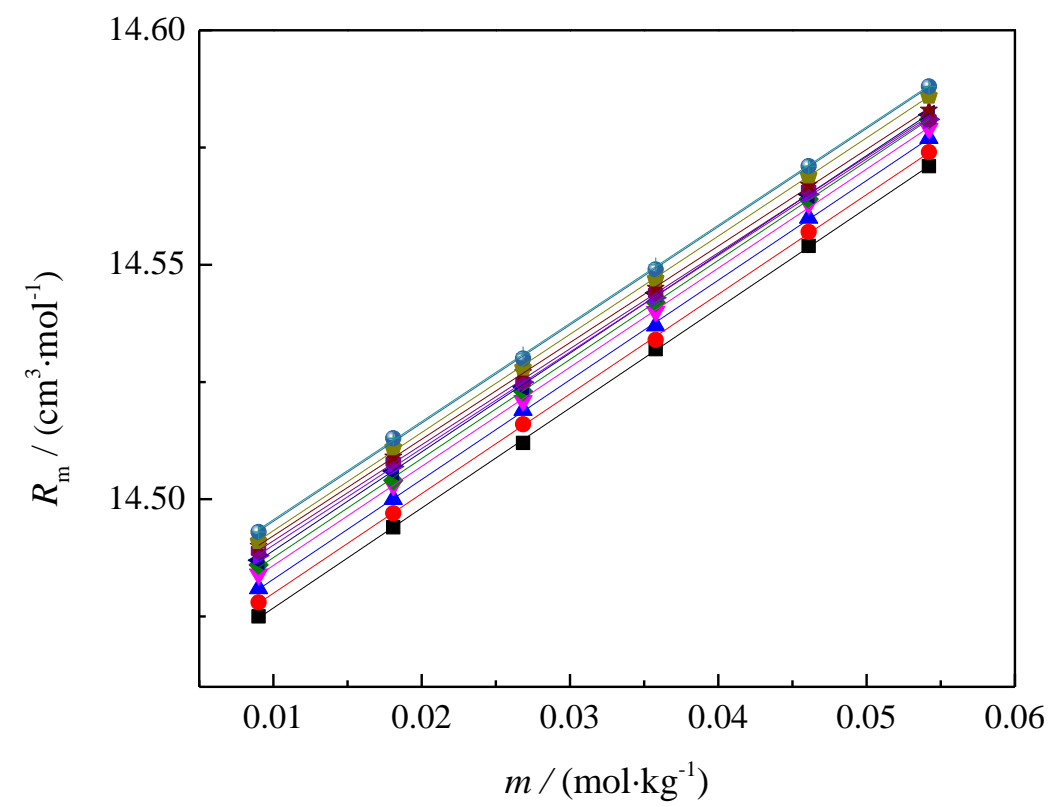

Figure S6. The dependence of the molar refractive index, $\left(R_{\mathrm{m}}\right)$, on caffeine molality, $(\mathrm{m})$, in caffeine + ethylene glycol solutions at different

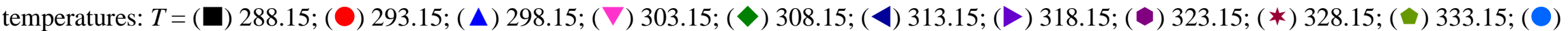
338.15 and $(+) 343.15 \mathrm{~K}$. The points are experimental values, while the lines represents fitts obtained by eq. S7. 